\title{
Titularidades y cátedras en Comunicación en España (2000-2019). Distribución, ritmos de promoción, transferencia entre universidades y endogamia
}

\author{
Professors and associate professors in the \\ Communication field in Spain (2000-2019). Distribution, \\ promotion rates, transfer between universities, \\ and endogamy
}

\author{
Rafael Repiso; Inmaculada Berlanga; Elías Said-Hung; Antonio Castillo-Esparcia
}

Cómo citar este artículo:

Repiso, Rafael; Berlanga, Inmaculada; Said-Hung, Elías; Castillo-Esparcia, Antonio (2020). "Titularidad y cátedras en Comunicación en España (2000-2019). Distribución, ritmos de promoción, transferencia entre universidades y endogamia". Profesional de la información, v. 29, n. 4, e290422.

https://doi.org/10.3145/epi.2020.jul.22

Artículo recibido el 27-03-2020 Aceptación definitiva: 05-06-2020
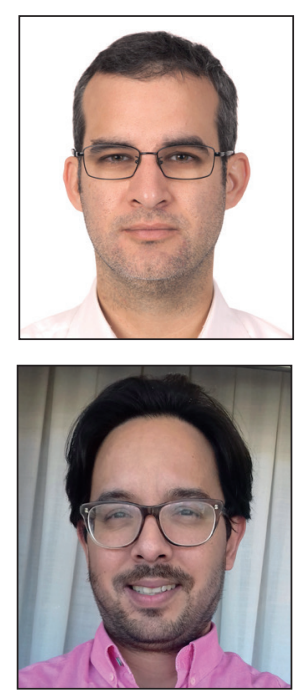

Rafael Repiso

https://orcid.org/0000-0002-2803-7505

Universidad Internacional de La Rioja Fac. de Humanidades y Ciencias Sociales Av. de la Paz, 137. 26006 Logroño, España rafael.repiso@gmail.com

Elías Said-Hung

https://orcid.org/0000-0002-0594-5906

Universidad Internacional de La Rioja

Facultad de Educación

Av. de la Paz, 137. 26006 Logroño, España elias.said@unir.net

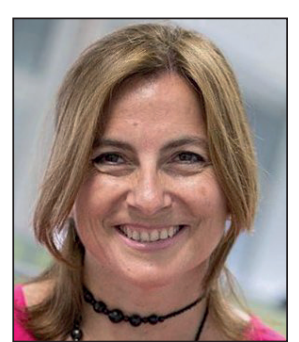

Inmaculada Berlanga $\square$ https://orcid.org/0000-0002-0135-624X

Universidad Internacional de la Rioja Fac. de Humanidades y Ciencias Sociales Av. de la Paz, 137. 26006 Logroño, España inmaculada.berlanga@unir.net

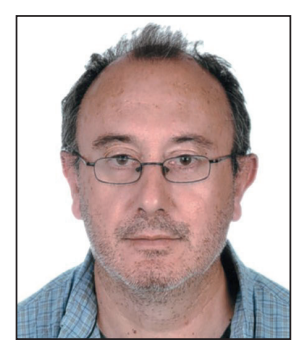

Antonio Castillo-Esparcia https://orcid.org/0000-0002-9751-8628

Universidad de Málaga

Facultad de Comunicación e Instituto de Investigación en Relaciones Públicas Campus de Teatinos, $\mathrm{s} / \mathrm{n}$. 29071 Málaga, España acastilloe@uma.es

\section{Resumen}

Este trabajo es un análisis estadístico descriptivo de la carrera académica de los titulares y catedráticos (funcionarios públicos) del área de Comunicación en España en el periodo 2000-2019. Se estudian tres fenómenos relacionados con la carrera académica en Comunicación. En primer lugar, la distribución de plazas por categoría, sexo y años. A continuación, los ritmos de promoción en esta área, a lo largo de todo el período y diferenciando entre los ritmos antes y después del sistema de acreditación de la Agencia Nacional de Evaluación de la Calidad y Acreditación (Aneca). Finalmente, los flujos entre las universidades donde se forma el claustro y las universidades públicas empleadoras de académicos, así como el fenómeno de la endogamia en el área. Con este fin, se han identificado en el Boletín oficial del Estado los nombramientos de titulares y catedráticos en las áreas de Periodismo y Comunicación Audiovisual y Publicidad. Un análisis a nivel colectivo y desagregado por universidades permite estudiar el rol de las universidades como instituciones que contratan y forman al claustro, y los flujos entre ellas. Los resultados muestran que la contratación de titulares y catedráticos de Comunicación en los 20 años estudiados se revela irregular en cuanto a distribución, paridad y adjudicación de plazas en el tiempo. A su vez, las sucesivas legislaciones han generado un sistema que reduce la movilidad de profesorado y favorece la endogamia. 


\title{
Palabras clave
}

Comunicación; Periodismo; Comunicación audiovisual; Publicidad; Carrera académica; Titularidades; Cátedras; Promoción académica; Endogamia; Universidad española; Género; Políticas científicas; Docencia universitaria; España.

\begin{abstract}
We present a descriptive statistical analysis of the academic career of professors and associate professors in the area of Communication in Spanish public universities during the period 2000-2019. Three phenomena related to academic careers in Communication are studied: first, the distribution of positions by category, sex, and years; second, promotion paths; third, the flows between universities, which show a phenomenon of endogamy. To this end, we identified the appointments of professors and associate professors in the areas of Journalism and Audiovisual Communication and Advertising in the Boletín oficial del Estado (the Spanish official gazette). Our analysis provides an understanding of the role of universities as institutions that hire and train faculty, and the flow of faculty among them. The results show that the recruitment of professors and associate professors in the 20 years studied is irregular in terms of distribution, parity, and allocation of positions over time. In turn, successive legislation has generated a system that reduces faculty mobility and favors endogamy.
\end{abstract}

\section{Keywords}

Communication; Audiovisual Communication; Journalism; Advertising; Academic career; Faculty; Tenure; Chairs; Academic promotion; Endogamy; Spanish university; Gender; Scientific policies; University teaching; Spain.

Financiación

Los costes de publicación y puesta en abierto de este artículo han sido cubiertos por la Universidad Internacional de La Rioja (UNIR).

Agradecimientos

A todos los que nos han ayudado a completar y verificar el listado de profesores funcionarios de Comunicación. Queremos agradecer también al profesor Blázquez Ochando por poner en disposición pública el set de datos de Teseo. Agradecer a los revisores anónimos por su aportación constructiva a este trabajo.

\section{Introducción}

El área de Comunicación en España destaca por el interés que suscita en el estudio de su corta vida: es una de las disciplinas que más se investiga a sí misma, por lo que ha originado decenas de artículos cuyo objeto de análisis ha sido principalmente la producción científica en el área a través de sus artículos, revistas científicas, tesis doctorales e incluso proyectos de investigación. Sin embargo, apenas hay trabajos científicos que se centren en un aspecto básico: la identificación y cuantificación de los miembros que componen el área -principales protagonistas y dinamizadores-, cómo se distribuyen por universidades, dónde se forman y dónde trabajan o cuáles son los tiempos habituales de promoción. Pocas obras analizan la población que conforma el área de Comunicación, apenas se ha investigado la transferencia entre universidades si no es en trabajos sobre tesis doctorales. La aproximación más cercana al análisis de la comunidad se ha hecho en investigaciones sobre producción científica y sus autores, pero sin identificar en primer lugar a la población, razón por la que se obvia una parte significativa de la comunidad: la que no investiga.

Fue la creación de las primeras facultades de Ciencias de la Información en el año 1971 y la posterior llegada de la democracia el fermento que explica la trayectoria y crecimiento de los estudios de Comunicación en España (De-Aguilera-Moyano, 1998; Jones, 1998). En 1971, el Decreto 2070 de 13 de agosto reguló los estudios de periodismo y demás medios de comunicación social en la universidad, facultando a las universidades a que pudieran solicitar la creación de facultades de Ciencias de la Información. Esos centros podrán impartir "enseñanzas correspondientes a Periodismo, Cinematografía, Televisión, Radiodifusión, y Publicidad", agrupándolas en tres secciones o ramas: Periodismo, Ciencias de la Imagen Visual y Auditiva, y Publicidad (Castillo; Xifra, 2006). Las primeras universidades en las que se impartieron esos estudios fueron la Universidad Complutense de Madrid, la Universitat Autònoma de Barcelona y la Universidad de Navarra. Hay que indicar que, aunque en el año 1984 se aprobaran para Comunicación tres áreas de conocimiento, a final del año sólo se incorporaron dos áreas específicas "Comunicación Audiovisual y Publicidad" y "Periodismo" (Cebrián, 2001). El gran crecimiento en número de estudios de Comunicación comenzó a finales de los años ochenta, crecimiento que se vio incrementado por la multiplicación de nuevas universidades públicas y privadas.

Los primeros estudios reflexivos sobre el área se dieron a final de los años ochenta y ya se percibía una fuerte tendencia por el estudio de su dimensión investigadora (De-Moragas-Spà, 1988; Caffarel-Serra; Domínguez; Romano, 1989; Gifreu, 1989). En total en los años noventa se publicó una docena de trabajos sobre Comunicación (Repiso, 2013) que en la siguiente década se multiplicaron, y actualmente es raro no ver un artículo de esta temática en cada número de las principales revistas de Comunicación españolas. Ante tal avalancha de trabajos, habría que resaltar por su impacto y continuidad en el área los trabajos de Carmen Caffarel y Manuel Martínez-Nicolás, que analizan en conjunto y con 
gran perspectiva temporal y temática el área de Comunicación en España (Caffarel-Serra; Domínguez; Romano, 1989; Martínez-Nicolás, 2006; Martínez Nicolás; Saperas-Lapiedra, 2011; Castillo-Esparcia; Carretón-Ballester, 2010; Caffarel-Serra; Ortega-Mohedano; Gaitán-Moya, 2017; 2018; Martínez-Nicolás; Saperas-Lapiedra; Carrasco-Campos, 2017; Martínez-Nicolás, 2020).

Si la principal transformación de la reciente universidad española ha sido su crecimiento, en las dos primeras décadas del siglo XXI, los principales elementos que han afectado a la carrera académica, objeto de este trabajo, han sido la sucesión de leyes universitarias, la inclusión en el sistema europeo de universidades (Bolonia) y una crisis económica que, entre otros efectos, paralizó durante años la contratación y promoción de profesores.

El siglo XXI se inició con la vigencia de la Ley orgánica 11/1983 de reforma universitaria (LRU) (España, 1983), ley que adaptó la institución universitaria a la Constitución de 1978. En sus artículos 37 y 38 se establecían los criterios para los concursos de titulares y catedráticos respectivamente, y que permitió la regulación del personal contratado (Puigdomènech, 1984). En el primer caso era necesario estar en posesión del título de doctor y una oposición pública, no pudiendo concursar quienes habían estado contratados durante más de dos años como ayudante en la universidad a la que corresponda dicha plaza. En el segundo caso, era necesario ser ya catedrático o profesor titular de universidad (o doctor y catedrático de escuela universitaria con tres años de antigüedad). Las comisiones se componían por cinco catedráticos, tres de ellos designados mediante sorteo por el Consejo de Universidades y dos por la propia universidad.

Dieciocho años después, durante el segundo gobierno de Aznar (PP), se promulgó la Ley orgánica 6/2001 de universidades (LOU) (España, 2001) que vino a sustituir a la $L R U$. En esa ocasión, el acceso a los cuerpos docentes universitarios quedó articulado en un doble proceso, a inspiración del sistema alemán (Martínez-Camacho, 2002), compuesto por una primera fase de habilitación nacional (Art. 57-62), que pretendía reducir la endogamia (Carbajo; Neira; Farrás, 2008) y una segunda fase de concursos de acceso internos a las universidades (Art. 63). El número de plazas convocadas se relacionaba con las plazas vacantes y la existencia de habilitados que no ocupasen plaza (Art. 3.2), por lo que era un procedimiento competitivo. El requisito para participar en las pruebas de habilitación de titular de universidad era ser doctor. En el caso de catedrático se requería, además, ser profesor titular de universidad o catedrático de escuela universitaria con tres años de antigüedad. Con todo, el Consejo de Coordinación Universitaria podría eximir de estos requisitos a los doctores con ocho años de antigüedad (Art. 5.1). Sólo con posterioridad a la selección por una universidad, al nombramiento y a la toma de posesión se adquiriría la condición de funcionario.

La LOU tuvo una vida corta. En el año 2007, durante el gobierno de Rodríguez Zapatero la LOU fue modificada por la Ley orgánica 4/2007 de 12 de abril (España, 2007). El acceso a los cuerpos de titulares y catedráticos se realiza por concurso para aquellos acreditados por la Agencia Nacional de Evaluación de la Calidad y Acreditación (Aneca) para tales figuras, creándose para este fin los programas PEP y Academia. La composición de las comisiones y el procedimiento de selección pasan a ser regulados por los estatutos de cada universidad, pero la realidad es que en la mayor parte de universidades el tribunal lo propone el departamento que solicita la plaza, con criterios divergentes y suele iniciarse a propuesta de un profesor acreditado del departamento que quiere que le reconozcan su reciente acreditación. En muchos casos la propuesta del tribunal que va a juzgar la plaza la hace el mismo solicitante (para perjuicio del resto de aspirantes), aunque también es posible que se realice a propuesta del conjunto del profesorado del departamento o a iniciativa de la presidencia de los miembros fijos del tribunal. Además permite el surgimiento de una práctica particularmente endogámica favoreciendo a los candidatos que dominan el idioma local (Carbajo; Neira; Farrás, 2008). Parece que la democracia ha traído a la universidad prácticas poco equitativas. Sólo hay que pensar que antes de la $L R U$ del año 1983 la selección de profesores era un proceso centralizado bajo una convocatoria única por área de conocimiento donde concurrían todos los profesores del Estado. Aquellos que más puntos sacaban elegían destino, una especie de MIR. De hecho, este cambio legislativo y sus inmediatas repercusiones fueron objeto de debate internacional.

La docencia universitaria es una de las profesiones que dedica más tiempo a procesos evaluativos: a ser evaluado (en su currículo, en sus trabajos, en sus proyectos) y a evaluar a otros, y lo hace por motivos que trascienden la propia evaluación: para mejorar las deficiencias detectadas en el trabajo universitario o para la toma de decisiones administrativas sobre los sujetos evaluados. En esencia, selección y promoción (De-la-Orden, 1990). Sin embargo, un elemento resultante que pone en duda la validez de los procesos evaluativos es la endogamia. Este trabajo también pretende cuantificar la endogamia en el campo de la Comunicación en los últimos 20 años para sus figuras principales -titulares y catedráticos- según universidades. La endogamia es una preocupación de actualidad en la universidad, aunque en centros de gran prestigio ha sido un aspecto muy cuidado desde hace más de un siglo. Por ejemplo, esto ya preocupaba al que fue el presidente de Harvard más longevo en su cargo, Charles W. Eliot (1869-1909), como se recoge en su obra "University administration" (Eliot, 1908). Y, pocas décadas después ya era un elemento de riesgo ampliamente estudiado en los sistemas universitarios estadounidenses. El experto en educación comparada Walter Crosby Eells se mostraba escandalizado en la década de los años 30 porque en Estados Unidos un tercio

Aunque el área de Comunicación en España es una de las disciplinas académicas que más se investiga a sí misma, apenas hay estudios sobre la identificación y cuantificación de los miembros que la componen 
de los profesores universitarios impartía docencia en las universidades en las que se habían formado, situación que se recrudecía en las universidades católicas donde ascendía a la mitad del claustro (Crosby-Eells; Cleveland, 1935).

El fenómeno endogámico en España comenzó en 1928 cuando se autorizaron los estudios de doctorados en cualquier universidad y se creó la figura del "padrino", predecesor del director de tesis (Repiso, 2013). Hasta ese momento, sólo la Universidad Central formaba doctores, de ahí que también fuese conocida como "La Docta". Pero como fenómeno de estudio, la endogamia universitaria se ha analizado tardíamente. Con el cambio de siglo fueron varios los autores que señalaron este aspecto, quizás con la intención de remarcar su gravedad e influir, infructuosamente, en el futuro desarrollo legislativo que se estaba gestando. Incluso la endogamia en España se hizo un hueco en las revistas Nature y Science (Camacho, 2001; Navarro; Rivero, 2001; Soler, 2001; Escartín, 2003; Muñoz-Pinedo et al., 2003; Secundo et al., 2015). Juan Pedro Camacho indicaba que la endogamia en España era reflejo de la falta de interés real por la universidad y la investigación y que, con una inversión mayor y responsable, que rindiese cuentas sobre resultados, las contrataciones serían para el personal más válido (Camacho, 2001; Martínez-Camacho, 2002) poniéndose coto a esta mala práctica.

El presente trabajo pretende identificar y caracterizar a los profesores funcionarios del área de Comunicación (titulares y catedráticos de universidad), pilares de la docencia y la investigación universitaria, que obtuvieron sus plazas entre los años 2000 y 2019 y, a través de esta muestra, estudiar tres fenómenos relacionados con la carrera académica en Comunicación:

- en primer lugar, la distribución de plazas por categoría, sexo y años;

- en segundo lugar, conocer los ritmos de promoción en el área de Comunicación desde la defensa de la tesis, la obtención de la titularidad hasta la cátedra; y,

- en tercer lugar, identificar los flujos entre las universidades donde se forma el claustro y las universidades públicas empleadoras de académicos. En este último apartado además estudiaremos el fenómeno de la endogamia en el área.

\section{Metodología}

Este trabajo es un análisis estadístico descriptivo de la carrera académica de los titulares y catedráticos (funcionarios públicos) del área de Comunicación en España para el periodo 2000-2019. Con este fin se han identificado en el Boletín oficial del Estado los nombramientos de titulares y catedráticos en las áreas de Periodismo y Comunicación Audiovisual, y Publicidad. Este análisis se realiza a nivel colectivo y desagregado por universidades, lo que permite estudiar el rol de las universidades, como instituciones que forman al claustro, que lo contrata, o ambas a la vez, así como estudiar los flujos entre universidades. Quedan excluidos del estudio las figuras de contratados laborales incorporadas recientemente al sistema catalán de universidades (profesores agregados y catedráticos laborales).

Para identificar a los profesores titulares se han llevado a cabo tres estrategias que se han complementado entre sí.

a) La descarga inicial se hizo a través del diseño de un script de Python, empleado para toda la información dispuesta en el portal del BOE, desde el 1 de enero de 2000 hasta el 31 de diciembre de 2019.

https://www.boe.es

Durante el proceso de elaboración del script se identificaron las taxonomías de organización de la información publicada en el $B O E$, tomando como referente de búsqueda la concurrencia del término "catedrático(a)", "catedráticos(as)", "titu$\operatorname{lar}(\mathrm{s})$ " en el título de cada BOE publicado en el período de tiempo estudiado. Los datos inicialmente recolectados, fueron revisados manualmente, con el fin de garantizar que la información extraída del portal del $B O E$ correspondiese con el objeto de estudio de este trabajo. Se excluyeron, durante el proceso de extracción antes mencionado, todas las publicaciones que tenían en sus títulos las palabras "convocan", "convoca", "corrige", "corrigen", "comisión". Se localizaron 248 contratos en Comunicación, entre titulares y catedráticos, pero el listado se mostró incompleto. La extracción permitió obtener información sobre el nombre completo, fecha de nombramiento, figura y área de conocimiento.

b) En segundo lugar, se hizo una búsqueda manual en el mismo periodo, incluyendo los mismos términos, pero reduciéndolos a las áreas de Periodismo y Comunicación Audiovisual y Publicidad, que incorporó 165 contratos de profesores que no se habían registrado previamente. Esta búsqueda suple a la primera, pues recupera los mismos contratos y algunos más, no obstante, se menciona la primera como curiosidad metodológica.

c) Por último, se compartió el listado en internet, foros IWETEL y Comunicación de RedIRIS (Repiso; Said-Hung, 2020); se publicitó en varios foros profesionales, y se solicitó a un conjunto de profesores puntuales que revisasen los listados en busca de ausencias o errores de sus centros. En este procedimiento se incorporaron 30 registros más, por lo que el registro de plazas definitivo asciende a 443 plazas.

Para identificar las tesis doctorales se ha utilizado la base de datos elaborada por Blázquez Ochando que cubre la base de datos Teseo hasta 2015 y en algunos casos se ha tenido que recurrir a la fuente original. En los casos en los que no se pudo localizar la tesis en Teseo se recurrió al catálogo colectivo de bibliotecas universitarias Rebiun. Se han podido identificar las tesis de 362 profesores de un total de 411 . Teseo es una base de datos cuyo objetivo es registrar las tesis producidas en las universidades españolas desde el año 1976 (Repiso; Torres-Salinas; Delgado López-Cózar, 2016), por lo que obviamente no se listan las tesis producidas en el extranjero, lo que significa que esta variable no ha sido estudiada. 
Finalmente se han encontrado 443 plazas, 349 de titulares y 94 de catedráticos de Comunicación para un total de 411 profesores. De estos profesores, sólo se han podido identificar las tesis de 362 de ellos (88\%).

Los elementos que se estudian son:

- Distribución por años y sexo de las plazas de titulares y catedráticos de Comunicación en el periodo 2000-2019 (figura 1). Pirámide de población, realizada con Microsoft Excel (Office 365).

- Ritmos de promoción académica en el área de Comunicación (figuras 2 y 3). Se hace para todo el periodo y también se diferencia entre los ritmos antes y después de la acreditación. Boxplots realizados con Tableau Public 2019 (Murray; Chabot, 2013).

- Distribución de plazas de titulares y catedráticos de Comunicación, formación doctoral del conjunto y tasas de endogamias (relación entre profesores contratados en el periodo y doctorados en la misma universidad) por universidades (tabla 2). La definición simple de endogamia es "la práctica de seleccionar a antiguos estudiantes de una institución como miembros de la facultad" (Crosby-Eells; Cleveland, 1935, p. 261), en este caso hemos limitado la formación al periodo doctoral.

- Red de transferencia entre universidades y endogamia (figura 3). Realizado con Pajek (Nooy; Mrvar; Batagelj, 2004).

Por último, indicamos que todos los datos en bruto del artículo están disponibles en Zenodo en acceso abierto (Repiso, 2020).

\section{Resultados}

En el periodo 2000-2019 se registran 94 plazas de catedráticos y 349 de titulares de universidad pública en Comunicación (Comunicación Audiovisual y Periodismo). En líneas generales, el número de titulares y catedráticos presenta una distribución desigual, por años y por sexo. En el caso de los titulares, en el periodo estudiado hay tres ciclos que registran un gran número de titularidades y dos periodos de poca promoción que suman seis años donde sólo se registran 18 titularidades (2004-2005 y 2012-2016) y tres periodos que aglutinan otros seis años donde se publican algo más de la mitad de las titularidades, 186 titularidades 52\% (2002-2003, 2008 y 2017-2019). En ambos grupos se da una mayor presencia de hombres, que es especialmente más llamativa en el de las plazas de catedráticos. En el caso de las titularidades, los hombres representan el 61\% del total, sin embargo, hay periodos como el año 2012, 2014, 2016, 2017, 2018 donde el número de mujeres titulares de universidad supera al de hombres. En el caso de las cátedras, las plazas masculinas ascienden al $80 \%$ del total, si bien se aprecia un cambio de disposición tanto en cátedras como en titularidades siendo menor la diferencia en años recientes.

En el periodo general el tiempo mínimo para obtener la cátedra desde que se obtiene el doctorado es de 7 años (caso de Álvarez Monzoncillo), el promedio es de 17 años (para los que lo logran), el mayor número de catedráticos la obtienen entre 14 y 21 años desde que se doctoran. Lógicamente, mucho menor es el plazo de obtención de la titularidad, que en

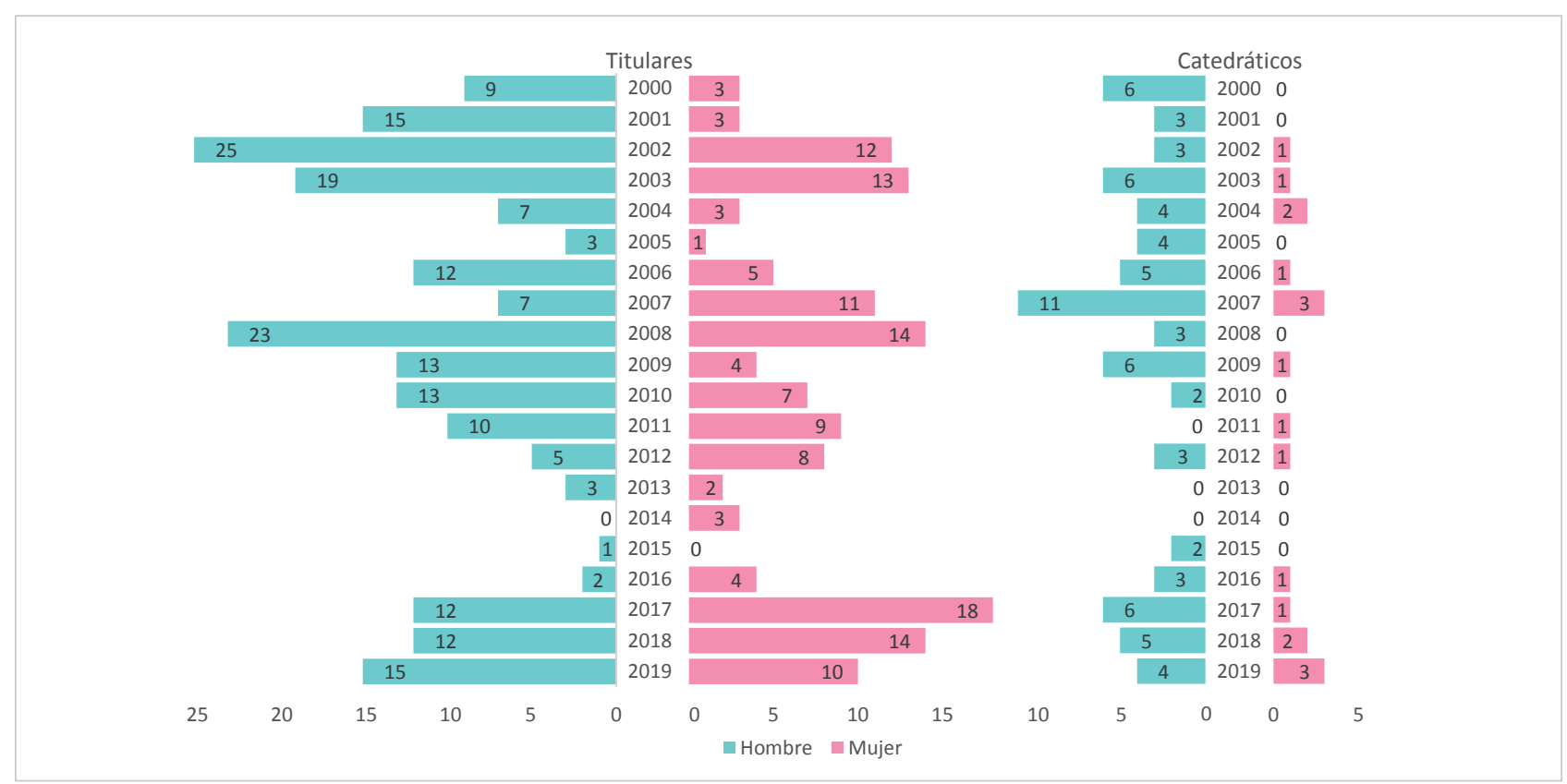

Figura 1. Pirámide de población de titulares y catedráticos de Comunicación en la Universidad Pública Española (2000-2019). 
algunos casos fue obtenida a los pocos meses de la defensa doctoral y en casos más extremos a los 34 años, aunque lo normal se sitúa entre los 5 y los 12 años. Una vez obtenida la plaza de titular, el tiempo de promoción hasta catedrático se encuentra entre 1 y 23 años, aunque el promedio está en 13 y lo normal es un periodo entre 7 y 15 años. Hay que tener en cuenta que los cálculos están restringidos en el caso de las titularidades a las de aquellos profesores que las consiguen y en las cátedras a los profesores que promocionan a catedrático.

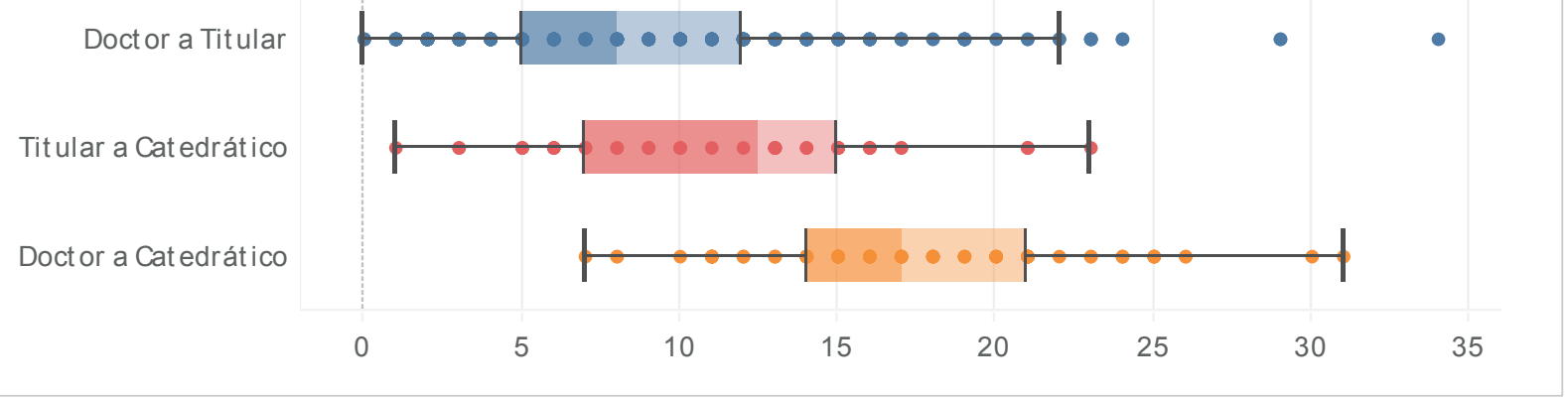

Figura 2. Ritmos de promoción académica. Desde el título de doctor a la cátedra. Comunicación. Periodo 2000-2019.

Si estudiamos los ritmos diferenciando dos periodos, dividiendo entre los que obtuvieron su acreditación antes y después de la vigencia del actual sistema de acreditación por Aneca, encontramos que los ritmos de promoción universitarios antes de la acreditación eran más rápidos: antes un profesor necesitaba una media de 5 años para obtener la plaza de titular desde que hacía la tesis, ahora con la acreditación se requerían 11 años. Para la cátedra sucede algo similar: el promedio antes de la acreditación era de 16 años para la obtención de la cátedra desde la defensa de la tesis, y sube a 20 años con la acreditación. Sin embargo, el mínimo para obtener ambas plazas no muestra una diferencia tan grande: el tiempo para obtener la titularidad en el sistema previo era de meses y con el actual es de 1 año; incluso el mínimo para ser catedrático con la acreditación es de 10 años mientras que en el anterior era de 11.

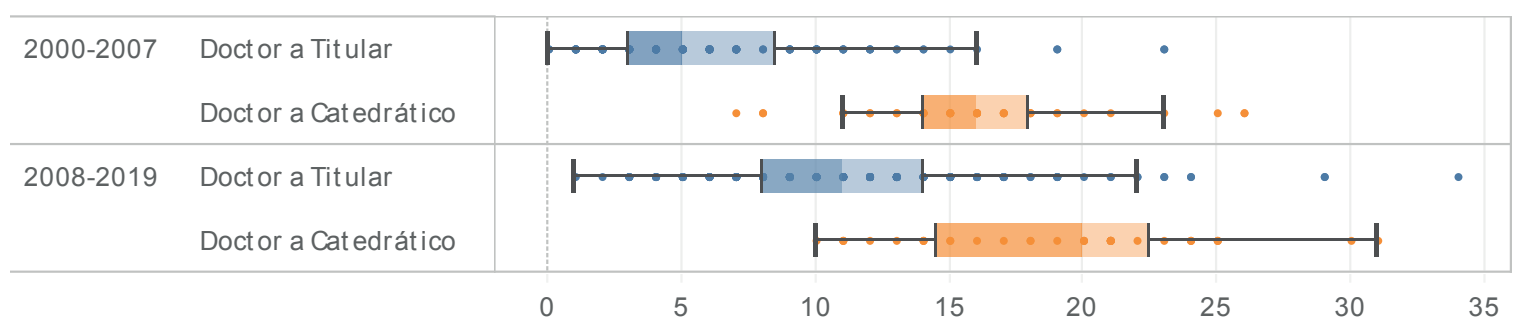

Figura 3. Ritmos de promoción académica diferenciado en dos periodos. Desde el título de doctor a la cátedra. Comunicación. Periodo $2000-2019$.

Tan sólo seis universidades han formado al 79\% de los profesores de Comunicación que han obtenido las plazas de titulares y catedráticos en los dos decenios estudiados (Univ. Complutense de Madrid, Univ. Autònoma de Barcelona, Univ. de Sevilla, Univ. de Valencia, Univ. de Málaga y Univ. del País Vasco). Todos estos centros registran tasas de endogamia superiores al 75\% con la excepción de la Universidad de Málaga. Hay que indicar que las universidades que han sacado un mayor número de plazas por catedráticos en relación con las de titulares son la Universitat Jaume I, Universidad del País Vasco y Universidad Complutense. Por el contrario, si excluimos a las universidades que han sacado menos de 7 plazas, serían las universidades de Alicante y de Murcia las que han obtenido un número relativo más reducido de catedráticos de Comunicación en estos dos últimos decenios.

La Universidad de La Laguna es la que muestra una tasa de endogamia mayor: todos los titulares y catedráticos que han obtenido su plaza en el periodo estudiado se habían formado en ella (en total tres). A poca distancia les siguen instituciones como la Universitat Autònoma de Barcelona, Universidad del País Vasco, Universidad Complutense y Universidad de Valencia con tasas de endogamia superiores al $80 \%$, todas ellas universidades con tradición en los estudios de Comunicación.

Para conocer mejor la influencia entre universidades a través del tránsito de doctores a plazas de titulares y catedráticos se ha generado la figura 4. La red social de transferencia entre universidades enriquece la información de la tabla 2, donde se aprecian los flujos entre universidades que forman docentes y las que los emplean. Igualmente se aprecia el tamaño del claustro de titulares y catedráticos obtenidos en el periodo 2000-2019.

Este trabajo es un análisis estadístico descriptivo de la carrera académica de los titulares y catedráticos del área de Comunicación en España para las dos primeras décadas del siglo XXI 
Tabla 2. Distribución por universidades del conjunto de titularidad y cátedra en Comunicación 2000-2019.

\begin{tabular}{|c|c|c|c|c|c|c|}
\hline Universidad & Titularidades & Cátedras & Total & $\begin{array}{c}\text { Tesis } \\
\text { dirigidas }\end{array}$ & $\begin{array}{l}\text { Profesores } \\
\text { exportados }\end{array}$ & $\begin{array}{c}\% \\
\text { endogamia* }\end{array}$ \\
\hline Univ. Complutense de Madrid & 37 & 23 & 60 & 131 & 85 & 82 \\
\hline Univ. Rey Juan Carlos & 43 & 8 & 51 & 0 & 0 & 0 \\
\hline Univ. Carlos III de Madrid & 31 & 6 & 37 & 0 & 0 & 0 \\
\hline Univ. de Sevilla & 32 & 5 & 37 & 38 & 10 & 76 \\
\hline Univ. de Málaga & 28 & 6 & 34 & 24 & 6 & 53 \\
\hline Univ. Autònoma de Barcelona & 20 & 6 & 26 & 44 & 24 & 87 \\
\hline Univ. de Valencia & 16 & 6 & 22 & 26 & 8 & 82 \\
\hline Univ. Pompeu Fabra & 15 & 6 & 21 & 8 & 6 & 13 \\
\hline Univ. de Alicante & 19 & 1 & 20 & 10 & 1 & 43 \\
\hline Univ. del País Vasco & 10 & 7 & 17 & 24 & 13 & 85 \\
\hline Univ. de Murcia & 15 & 1 & 16 & 5 & 2 & 20 \\
\hline Univ. Jaume I & 8 & 7 & 15 & 4 & 0 & 27 \\
\hline Univ. de Santiago de Compostela & 8 & 4 & 12 & 6 & 1 & 42 \\
\hline Univ. de Valladolid & 8 & 2 & 10 & 1 & 0 & 10 \\
\hline Univ. de Vigo & 8 & 2 & 10 & 5 & 1 & 40 \\
\hline Univ. Politécnica de Valencia & 8 & 2 & 10 & 8 & 2 & 60 \\
\hline Univ. Rovira i Virgili & 6 & 0 & 6 & 0 & 0 & 0 \\
\hline Univ. de A Coruña & 5 & 0 & 5 & 2 & 1 & 25 \\
\hline Univ. de Salamanca & 4 & 1 & 5 & 4 & 3 & 20 \\
\hline Univ. Miguel Hernández & 4 & 1 & 5 & 0 & 0 & 0 \\
\hline Univ. de Castilla-La Mancha & 4 & 0 & 4 & 0 & 0 & 0 \\
\hline Univ. de Burgos & 3 & 0 & 3 & 0 & 0 & 0 \\
\hline Univ. de Extremadura & 3 & 0 & 3 & 1 & 1 & 0 \\
\hline Univ. de La Laguna & 3 & 0 & 3 & 9 & 5 & 100 \\
\hline Univ. de Cádiz & 2 & 0 & 2 & 0 & 0 & 0 \\
\hline Univ. de Girona & 2 & 0 & 2 & 1 & 0 & 50 \\
\hline Univ. de Granada & 2 & 0 & 2 & 0 & 0 & 0 \\
\hline Univ. de Zaragoza & 2 & 0 & 2 & 0 & 0 & 0 \\
\hline Univ. Autónoma de Madrid & 1 & 0 & 1 & 5 & 4 & 100 \\
\hline Univ. de Alcalá & 1 & 0 & 1 & 0 & 0 & 0 \\
\hline Univ. de Barcelona & 1 & 0 & 1 & 6 & 6 & 0 \\
\hline
\end{tabular}

* Las tesis dirigidas y los profesores exportados (profesores formados y que ejercen de titulares o catedráticos en otras instituciones) se refieren al conjunto de profesores analizados, no a profesores externos al estudio.

La red (figura 4) muestra también cómo hay una serie de universidades que destacan por su papel formativo de profesores que posteriormente trabajarán en otros centros. Entre ellas destacan las universidades históricas en estudios de Comunicación: Universidad Complutense, Universitat Autònoma de Barcelona y Universidad de Navarra. Curiosamente, es la Universidad de Navarra el único centro que no posee titulares y catedráticos funcionarios (al ser privada) y que sin embargo ocupa una posición relevante en la estructura de la Comunicación académica al haber formado a 15 profesores que obtienen 18 plazas.

La proximidad geográfica se muestra como el principal factor relacional. La Universidad Complutense es la que más profesores de Comunicación transfiere a otros centros y tiene especial incidencia en las universidades Rey Juan Carlos y Carlos III de Madrid. Pero en realidad la Complutense parece tener una presencia destacada en las principales facultades de Comunicación de España salvo en las catalanas donde es la Universitat Autònoma de Barcelona la que articula la formación de los titulares y catedráticos contratados en 2000-19. En relación con las universidades receptoras es la Carlos III de Madrid en la que obtienen contratos de titulares y catedráticos profesores de una mayor variedad de universidades (Universidad Complutense de Madrid [11], Universidad de La Laguna [4], Universitat Autònoma de Barcelona [4], Universidad de Sevilla [2], Universidad Autónoma de Madrid [2], Universidad de Navarra [2], Universidad del País Vasco [2]). Por el contrario, los contratos de titulares y catedráticos de la Universidad Rey Juan Carlos parecen limitarse mayoritariamente a profesores formados en la Universidad Complutense (38 contratos) y en menor medida las Universidades de Salamanca y Navarra (2 contratos cada una) y otras seis universidades con un contrato cada una. 


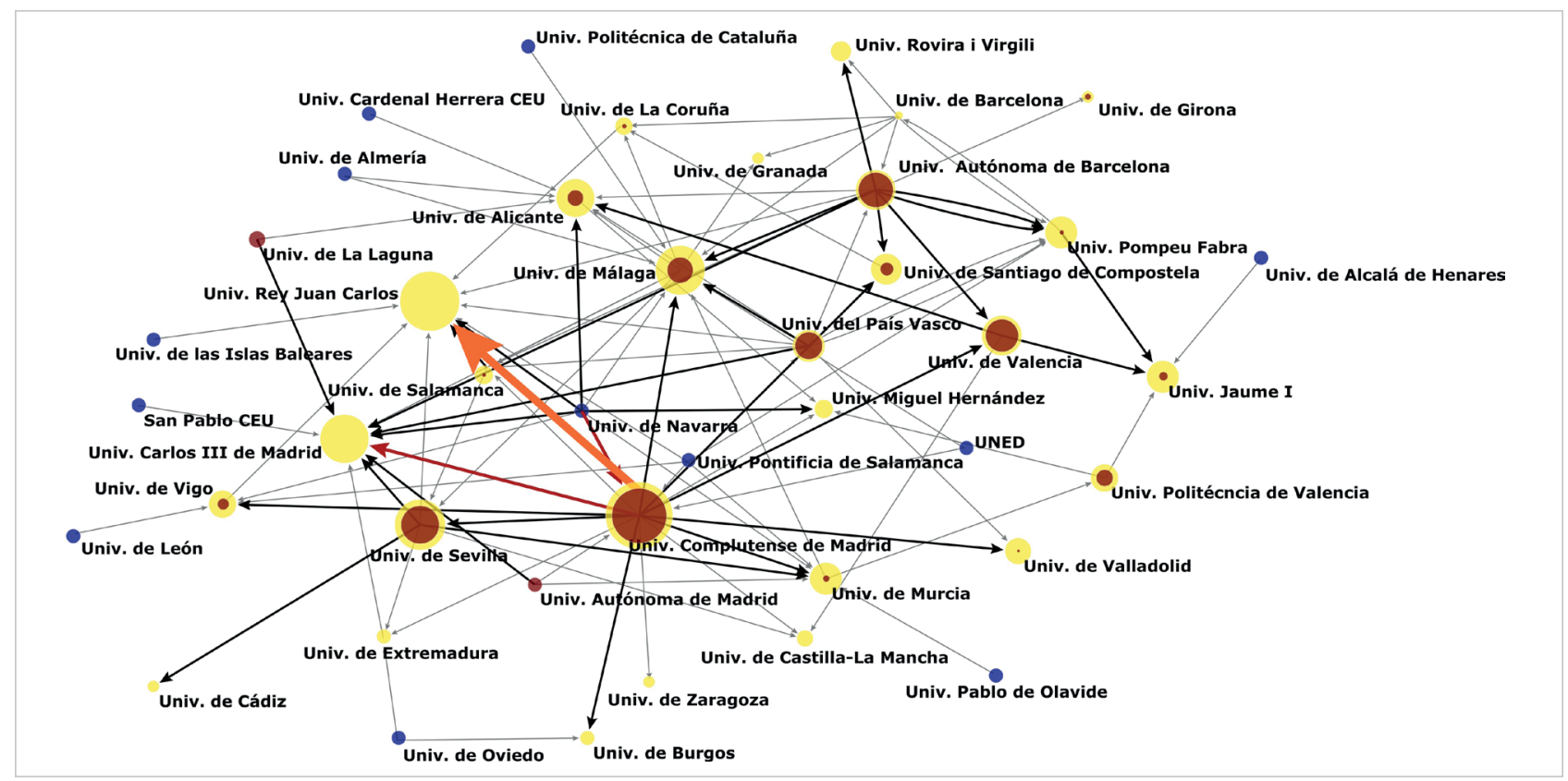

Figura 4. Transferencia de doctores a universidades y endogamia en Comunicación (titulares y catedráticos 2000-19).

Nota: El tamaño representa el número de contratos de titular y catedráticos en cada universidad. El amarillo indica el número de profesores formados en otras universidades, el rojo los formados en la propia universidad (endogamia). En color violeta las universidades que sólo forman profesores. El color y grosor de los enlaces representan el valor del "trasvase" de la universidad formadora a la universidad donde el profesor ejercita su labor.

\section{Discusión y conclusiones}

El primer cambio significativo de la universidad española en el siglo XXI fue la Ley Orgánica 6/2001, de 21 de diciembre, de universidades ( $L O U$ ) que legisló sobre la articulación de la universidad española en torno a la Administración General del Estado y las Comunidades Autónomas y estableció la creación de una agencia de acreditación tanto para las universidades y sus títulos como para los profesores (Agencia Nacional de Evaluación de la Calidad y Acreditación, Aneca), modificada por la Ley orgánica 4/2007 de 12 de abril. El objetivo declarado de ambas fue aumentar la excelencia de la universidad española en sus diferentes dimensiones y prepararla para su ingreso en el Sistema Universitario Europeo. Por ello, en menos de 9 años hemos tenido tres procesos evaluativos:

- el anterior a la LOU,

- la habilitación al comienzo de la aplicación de la LOU (copiado del sistema alemán (Martínez-Camacho, 2002), y

- desde el año 2008 la acreditación de Aneca, sistema que a su vez ha tenido dos etapas diferenciadas y separadas por unos años en los que, debido a la crisis económica, el estado anuló la contratación y redujo al mínimo la reposición del profesorado.

Todo este cambio legislativo ha hecho que la contratación de profesores titulares haya variado en el tiempo, en número y en requisitos. Hay que indicar que en relación a la endogamia, aspecto estudiado en este trabajo, ya se avisaba que la nueva legislación que acompañaba la creación de la Aneca y dejaba en manos de las universidades la contratación iba a convertir la endogamia en un mal crónico (Rocca, 2007).

La contratación de titulares y catedráticos de Comunicación entre los años 2000 y 2019 se muestra irregular en muchos sentidos. El número de plazas de titulares es 3,7 veces superior a las de catedráticos. Las mujeres representan un $36,5 \%$ del total de contratos, un $41 \%$ en las titularidades frente a un $19 \%$ en las cátedras; sin embargo, la tendencia muestra cómo cada vez hay una mayor presencia de éstas en ambas figuras, hasta el punto de que en titularidades hay años donde las mujeres han ganado la mayoría de los contratos. El mayor desajuste observado tiene que ver con la evolución de la adjudicación de plazas a lo largo del tiempo, siendo dos periodos los que concentran un mayor número de plazas: el periodo previo a la habilitación donde apenas existían controles y requisitos evaluativos (2000-2003) y el de 2017-2019 cuando se permitió la contratación de nuevo. En el informe EU2015 (Tarrach et al., 2011) se decía que la segunda década del siglo XXI sería una gran oportunidad para las universidades españolas, para consolidarse en el Espacio Europeo: aprovechando la jubilación de $1 / 3$ de los profesores se deberían contratar profesores investigadores con experiencia internacional, así como reducir las ratios de alumno / profesor. Sin entrar en las contrataciones, lo que sabemos es que, en lugar de aumentar las ratios, la contratación ha decaído; además muestra una gran improvisación y vista la gran endogamia se duda de que se haya avanzado en la internacionalización del claustro. Queda por tanto estudiar el nivel científico productivo de los profesores contratados.

La contratación de titulares y catedráticos de Comunicación en las dos primeras décadas del siglo XXI resulta irregular en cuanto a distribución, paridad y adjudicación de plazas en el tiempo 
Es evidente que la mayor desigualdad entre hombres y mujeres se presenta en las cátedras, sin embargo, esta desigualdad es casi la misma que se da en el sistema español, donde en el año 2016 las mujeres representan el 21,3\% de las cátedras y el 40,3\% de las titularidades (Ministerio de Ciencia, Innovación y Universidades, 2019). En un caso del año 2006 aplicado a la universidad australiana se aprecia algo parecido (Winchester et al., 2006). Los datos indicaban que no existía paridad, pero se estaban acercando progresivamente: en las figuras superiores había una desproporción significativa y similar a la encontrada en el presente trabajo, sólo un $16 \%$ de los profesores de más nivel eran mujeres (frente a un $20 \%$ en el presente trabajo). Habría que discutir si las medidas de igualdad tomadas en España y relacionadas con la paridad en la representación de tribunales y comisiones son realmente eficientes o, todo lo contrario, ya que obligan a las académicas a multiplicar su presencia en actos que poco o nada ayudan en la promoción personal detrayendo mucho tiempo de sus tareas profesionales y personales.

Un efecto directo sobre la variabilidad de la contratación es la alteración de los ritmos de promoción en la universidad. En líneas generales para obtener la titularidad en el periodo estudiado se tarda un promedio de 8 años y el promedio para obtener la cátedra asciende a los 17 años desde la defensa de la tesis (los que la consiguen), si bien, hay cierta variación entre los ritmos de promoción antes y después de Aneca. Antes del sistema de acreditación se tardaba un promedio de 5 años en pasar a titular desde la defensa de la tesis (alguno lo consiguió a los pocos meses); con la acreditación el promedio aumenta a 11 años. En el caso de los catedráticos, antes de Aneca estos obtenían su plaza después de 16 años como doctor; después de Aneca el promedio asciende a 21 años. Esos 5/6 años pueden achacarse al aumento de requisitos académicos que llevan implícitos los criterios de la acreditación, así como los años de crisis en los que no ha habido contrataciones, lo que ha alargado la promoción universitaria retrasando las carreras académicas y envejeciendo al claustro. Un ejemplo de cómo los criterios de Aneca marcan pautas es el periodo mínimo que por normativa se debe cumplir para conseguir la acreditación a catedrático: 8 años desde la defensa de la tesis doctoral. Pero lógicamente, lo más costoso es conseguir los méritos que Aneca exige pues obtenerlos llevan aparejada una periodización. En el caso de Comunicación, 6 años de como IP (investigador principal) de proyectos de investigación, 4 proyectos como miembro (12 años), 3 sexenios de investigación (18 años), 11 años a tiempo completo de experiencia docente y la dirección de tesis que está entre 3 y 4 años según la normativa actual.

Las universidades que han formado a la mayor parte de los profesores que obtuvieron su titularidad y cátedra en el periodo 2000-2019 son las universidades históricas, (Complutense de Madrid y Autònoma de Barcelona), incluso la Universidad de Navarra, aun estando fuera del sistema público de universidades. Lógicamente, son las universidades que más han crecido (Universidad Rey Juan Carlos y Universidad Carlos III de Madrid); y aquellas que tenían un claustro mayor (Universidad Complutense, Universitat Autònoma de Barcelona, Universidad de Valencia, Universidad de Málaga...), las que han contratado a más profesores funcionarios de Comunicación. Un matiz relevante y significativo es la endogamia, donde se aprecia que los mayores niveles se concentran en las universidades históricas, fenómeno ya descrito en otros trabajos (Tavares et al., 2015) o en la Universidad de La Laguna donde el 100\% de las plazas de titulares y catedráticos fueron ganadas por profesores formados en el mismo centro (tres en total), por lo que la insularidad se ha identificado en la bibliografía como una dimensión influyente en la endogamia (Tavares; Lança; Amaral, 2017). La endogamia es un elemento que la comunidad del área debería estudiar en profundidad, pues muchos expertos como Luis F. Rull la asocian directamente con la reducción de la calidad docente e investigadora en España (Rocca, 2007). Por otra parte, sobra decir que las universidades recién creadas (Universidades Rey Juan Carlos y Carlos III de Madrid) tienen una tasa de endogamia cero, de momento.

Las sucesivas legislaciones universitarias han concentrado las contrataciones en las mismas universidades, lo que ha generado un sistema que reduce la movilidad de profesorado y favorece la endogamia. Pensemos que con las dificultades de comunicación de la época, nuestro nobel Ramón y Cajal ganó varias oposiciones en varios lugares de España (y también perdió una plaza de Granada) y fue profesor en las facultades de Medicina de Zaragoza, Valencia y Barcelona antes de recaer en la San Carlos de Madrid (Ramón y Cajal, 1995). Los resultados de endogamia obtenidos en el área de Comunicación muestran un gran parecido al conjunto español. El informe de 2008 sobre transparencia de la Fundación CYD calcula la endogamia española en torno al 68,8\% y en algunas comunidades como País Vasco o Canarias asciende al 91\% (Fundación CYD, 2018). Es llamativo que en un mundo donde las distancias se han reducido tanto por las infraestructuras y los medios de transporte y las universidades se han multiplicado en número los profesores universitarios cada vez tengan menor movilidad.

El presente estudio abre la puerta a seguir investigando en la promoción académica, su importancia y las repercusiones que tiene en el área; e igualmente es una llamada a profundizar en otro aspecto fundamental: la endogamia y sus consecuencias. En futuros trabajos se abordará la vinculación entre la promoción universitaria y la endogamia con otras variables como el sexo, la edad, producción y repercusión científica o la especialización temática.

\section{Referencias}

Caffarel-Serra, Carmen; Domínguez, Milagros; Romano, Vicente (1989). “El estado de la investigación de Comunicación en España (1978-1987)". El estado de la investigación de Comunicación en España (1978-1987), n. 3, pp. 45-57.

Caffarel-Serra, Carmen; Ortega-Mohedano, Félix; Gaitán-Moya, Juan-Antonio (2017). “Investigación en comunicación en la universidad española en el período 2007-2014". El profesional de la información, v. 26, n. 2, pp. 218-227.

https://doi.org/10.3145/epi.2017.mar.08 
Caffarel-Serra, Carmen; Ortega-Mohedano, Félix; Gaitán-Moya, Juan-Antonio (2018). “La investigación en comunicación en España: Debilidades, amenazas, fortalezas y oportunidades”. Comunicar, v. 26, n. 56, pp. 61-70. https://doi.org/10.3916/C56-2018-06

Camacho, Juan-Pedro-María (2001). "Investment is the best cure for inbreeding”. Nature, v. 413, n. 6852, p. 107. https://doi.org/10.1038/35093265

Carbajo, Rodrigo J.; Neira, José-Luis; Farràs, Rosa (2008). "Spain should implement a model that's known to work". Nature, v. 453, n. 1, pp. 26-27.

https://doi.org/10.1038/453026c

Castillo-Esparcia, Antonio; Xifra, Jordi (2006): "Investigación bibliométrica de las tesis doctorales españolas sobre relaciones públicas (1965-2005)". Anàlisi, n. 34, pp. 141-161.

https://ddd.uab.cat/record/15568

https://www.raco.cat/index.php/Analisi/article/view/55449

Castillo-Esparcia, Antonio; Carretón-Ballester, Mari-Carmen (2010): “Investigación en Comunicación. Estudio bibliométrico de las revistas de Comunicación en España”. Comunicación y sociedad, v. XXIII, n. 2, pp. 289-327. http://rua.ua.es/dspace/handle/10045/22678

Cebrián-Herreros, Mariano (2001). “La edición en el sector audiovisual”. Zer. Revista de estudios de comunicación, v. 6, n. 11. https://www.ehu.eus/ojs/index.php/Zer/article/view/6070/5752

Crosby-Eells, Walter; Cleveland, Austin-Carl (1935). "Faculty inbreeding". The journal of higher education, v. 70, n. 5, pp. 579-588.

https://doi.org/10.1080/00221546.1999.11780790

De-Aguilera-Moyano, Miguel (1998). “La investigación sobre comunicación en España: una visión panorámica”. Comunicación y cultura, n. 4, pp. 5-12. https://doi.org/10.1174/113839598322025919

De-la-Orden-Hoz, Arturo (1990). "Evaluación, selección y promoción del profesor universitario". Revista complutense de educación, v. 1, n. 1, pp. 11-29.

https://revistas.ucm.es/index.php/RCED/article/view/RCED9090130011A

De-Moragas-Spà, Miguel (1988). “Los estudios sobre comunicación y nuevas tecnologías en España: indicaciones sobre sus antecedentes y estado actual”. Cuadernos de investigación en comunicación, n. 1, pp. 11-19.

De-Nooy, Wouter; Mrvar, Andrej; Batagelj, Vladimir (2004). Exploratory social network analysis with Pajek. Cambridge: Cambridge University Press. ISBN: 9781107002388

Eliot, Charles-William (1908). University administration. Boston: Houghton Mifflin.

Escartín, Javier (2003). "Differing views on science in Spain". Science, v. 300, n. 5616, pp. 51-52. https://doi.org/10.1126/science.300.5616.51c

España (1983). "Ley orgánica 11/1983, de 25 de agosto, de reforma universitaria”. BOE, n. 209, 1 de septiembre. https://www.boe.es/eli/es/lo/1983/08/25/11

España (2001). “Ley orgánica 6/2001, de 21 de diciembre, de universidades”. BOE, n. 307, 24 de diciembre. https://www.boe.es/eli/es/lo/2001/12/21/6

España (2007). “Ley orgánica 4/2007, de 12 de abril, por la que se modifica la Ley orgánica 6/2001, de 21 de diciembre, de universidades". BOE, n. 89. 13 de abril.

https://www.boe.es/eli/es/lo/2007/04/12/4

Fundación CYD (2018). "Informe CYD 2018".

https://www.fundacioncyd.org/publicaciones-cyd/informe-cyd-2018

Gifreu, Josep (1989). "La investigació de la comunicació a Catalunya: assaig de periodització". Anàlisi: quaderns de comunicació i cultura, n. 12, pp. 9-65. https://ddd.uab.cat/pub/analisi/02112175n12/02112175n12p9.pdf

Jones, Daniel E. (1998). “Investigación sobre comunicación en España: evolución y perspectivas”. Zer, v. 3, n. 5, pp. $13-51$. https://ddd.uab.cat/record/25038

Martínez-Camacho, Juan-Pedro (2002). “Endogamia universitaria: 'diálogos de sordos'”. Apuntes de ciencia y tecnología, n. 5, pp. 12-15.

Martínez-Nicolás, Manuel (2020). "Communication research in Spain (1985-2015). Institutional context, academic community and scientific production". Revista latina de comunicación social, n. 75, pp. 383-414.

https://doi.org/10.4185/RLCS-2020-1432 
Martínez-Nicolás, Manuel; Saperas-Lapiedra, Enric (2011). “La investigación sobre Comunicación en España (19982007). Análisis de los artículos publicados en revistas científicas”. Revista latina de comunicación social, n. 66, pp. 101-29. http://www.revistalatinacs.org/11/art/926_Vicalvaro/05_Nicolas.html

Martínez-Nicolás, Manuel; Saperas-Lapiedra, Enric; Carrasco-Campos, Ángel (2017). "La investigación sobre periodismo en España. Análisis de los trabajos publicados en revistas científicas españolas en los últimos 25 años (1990-2014)". Comunicación y sociedad, v. 30, n. 4, pp. 149-166.

https://doi.org/10.15581/003.30.3.149-166

Ministerio de Ciencia, Innovación y Universidades (2019). Datos y cifras del Sistema Universitario Español. Publicación 2018-2019, Madrid: Ministerio de Ciencia, Innovación y Universidades. e-NIPO: 692-19-015-9

https://www.ciencia.gob.es/stfIs/MICINN/Universidades/Ficheros/Estadisticas/datos-y-cifras-sue-2018-19.pdf

Muñoz-Pinedo, Cristina; Del-Pozo, Miguel A.; Gálvez, José A.; Moreno, Eva-María; Buceta-Fernández, Javier; García-Sánchez, Marta; Valdivieso, José-María; Bascones, Elena (2003). "Spanish scientists working abroad”. Science, v. 300, n. 5616, p. 51. https://doi.org/10.1126/science.300.5616.51b

Murray, Dan; Chabot, Christian (2013). Tableau your data!: Fast and easy visual analysis with Tableau software, Indianapolis: Wiley \& Sons. ISBN: 9781118612040

Navarro, Arcadio; Rivero, Ana (2001). "High rate of inbreeding in Spanish universities". Nature, v. 410, n. 14. https://doi.org/10.1038/35065259

Puigdomènech, Pedro (1984). "Reforms beginning to work". Nature, v. 312, n. 8. https://doi.org/10.1038/312008a0

Ramón y Cajal, Santiago (1995). Recuerdos de mi vida: historia de mi labor científica. Madrid: Alianza Universidad. ISBN: 9788420622903

Repiso, Rafael (2013). La investigación en Televisión, Cine, Radio y Fotografía en España: Análisis bibliométrico y de redes sociales de la estructura cientifica. Tesis doctoral, Universidad de Granada.

https://www.slideshare.net/rrepiso/tesisrepiso

Repiso, Rafael (2020). Titulares y catedráticos en Comunicación en España (2000-2019) [Data set]. Profesional de la información. Zenodo.

http://doi.org/10.5281/zenodo.3957203

Repiso, Rafael; Said-Hung, Elías (2020). Titulares y catedráticos de comunicación en universidades españolas (version 1) [Data set]. Zenodo.

http://doi.org/10.5281/zenodo.3638224

Repiso, Rafael; Torres-Salinas, Daniel; Delgado-López-Cózar, Emilio (2016). "Análisis de la relación entre disciplinas a través del uso de tesis doctorales. El caso de Televisión, Radio, Cine y Fotografía en España". Revista latina de comunicación social, v. 71, pp. 874-890.

https://doi.org/10.4185/RLCS-2016-1125

Rocca, Francis X. (2007). “In Spain, inbreeding threatens academe”. Chronicle of higher education, v. 53, n. 22, pp. $162-169$. https://doi.org/10.17323/1814-9545-2009-1-162-169

Secundo, Giustina; Elena-Pérez, Susana; Martinaitis, Žilvinas; Leitner, Karl-Heinz (2015). “An intellectual capital maturity model (ICMM) to improve strategic management in European universities". Journal of intellectual capital, v. 16, n. 2, pp. 419-442. https://doi.org/10.1108/JIC-06-2014-0072

Soler, Manuel (2001). "How inbreeding affects productivity in Europe". Nature, v. 411, n. 132.

https://doi.org/10.1038/35075637

Tarrach, Rolf; Egron-Polak, Eva; De-Maret, Pierre; Rapp, Jean-Marc; Salmi, Jamil (2011). Audacia para llegar lejos: universidades fuertes para la España del mañana. Informe de la Comisión de Expertos Internacionales de la EU2015. https://www.usc.es/export9/sites/webinstitucional/gl/web/descargas/informe-finaleu2015.pdf

Tavares, Orlanda; Cardoso, Sónia; Carvalho, Teresa; Sousa, Sofia-Branco; Santiago, Rui (2015). “Academic inbreeding in the Portuguese academia". Higher education, v. 69, n. 6, pp. 991-1006.

https://doi.org/10.1007/s10734-014-9818-x

Tavares, Orlanda; Lança, Vasco; Amaral, Alberto (2017). "Academic inbreeding in Portugal: Does insularity play a role?". Higher education policy, v. 30, n. 3, pp. 381-399.

https://doi.org/10.1057/s41307-016-0029-1

Winchester, Hilary; Lorenzo, Shard; Browning, Lyn; Chesterman, Colleen (2006). “Academic women's promotions in Australian universities". Employee relations, v. 28, n. 6, pp. 505-522.

https://doi.org/10.1108/01425450610704461 